\title{
Surgical Treatment of Congenital Radial Head Luxation in a Cat by Ostectomy of Radial Head
}

\author{
Pauline Verdese $^{1} \quad$ Sara Manfredini $^{1} \quad$ Luca Formaggini $^{1}$ \\ ${ }^{1}$ Clinica Veterinaria Lago Maggiore, Dormelletto, Novara, Italy \\ Address for correspondence Pauline Verdese, DVM, Clinica \\ Veterinaria Lago Maggiore, Corso Camillo Cavour 3, 28040 \\ VCOT Open 2022;5:e1-e5. \\ Dormelletto, Novara, Italy (e-mail: pauline.verdese@gmail.com).
}

\begin{abstract}
Keywords

- bone development

- limb deformity

- orthopaedic surgery

Congenital radial head luxation was diagnosed in a 7-month-old, neutered female cat that presented a right forelimb lameness. Clinical and radiographic examination revealed a caudolateral luxation of the right radial head, which was treated by radial head ostectomy. For months, postoperative radiographic recheck documented remodelling of the radial head, without humero-radial conflict, and clinical examination revealed resolution of lameness and restoring of the normal elbow range of motion. To the authors' knowledge, this is the first case report of a congenital radial head luxation in a caudolateral direction in a cat that was successfully managed surgically with radial head ostectomy.
\end{abstract}

\section{Introduction}

The feline elbow is a composite joint made up of the humeroradial, humero-ulnar and proximal radio-ulnar articulations. Elbow luxation has been classified as traumatic, congenital or developmental. ${ }^{1}$ Congenital elbow luxation (CEL) occurs infrequently, but it has been reported to be part of 17 to $20 \%$ of non-fracture elbow lameness ${ }^{2}$ in dogs.

The congenital or developmental forms of luxations have been sub-classified according to variations in anatomy. Humero-radial luxation (type 1 ) occurs when the radial head is displaced, and the humero-radial and proximo radio-ulnar articulations are disrupted. Humero-ulnar luxation (type 2) occurs when ulnar displacement results in disruption of the humero-ulnar and proximal radio-ulnar articulations, and combined humero-radial and humeroulnar luxation (type 3) occurs when both the radius and ulna are displaced as a unit, causing disruptions of the humero-radial and humero-ulnar articulations.

Data reporting CEL in cats are still lacking in the literature, while several studies on this pathology in dogs have been published.

The most frequent atraumatic canine elbow luxation is the radial head luxation (type I). ${ }^{3,5}$

received

July 5, 2021

accepted after revision

November 22, 2021
The aetiology of canine radial head luxation is still debated, but it seems to have a hereditary basis suggested by the frequency of bilateral involvement, the occurrence of congenital luxation of the radial head in more than one littermate, the multiple anomalies in some individuals and the embryonic failure of the elbow joint that does not develop properly. ${ }^{6-8}$

The hypoplasia or aplasia of the annular ligament, medial coronoid process or anconeal process has been hypothesized as the underlying cause of the pathophysiological congenital luxation of the radius. ${ }^{9,10}$ However, there is a lack of conclusive information about the possible etiologies. ${ }^{10,11}$

Clinical signs of type I CEL are more frequently observed in dogs that are around 3 and 4 months of age and in mediumlarge breeds. ${ }^{3,11,12}$ Nevertheless, there are recent reports of CEL occurring also in small breeds. ${ }^{13-17}$

Affected puppies may present slight external rotation of the antebrachium, a valgus deformity at the carpus and mild lameness. On physical examination, the radial head can be palpated lateral to the joint and the range of motion is limited in flexion and extension. Muscle atrophy, pain and crepitus often develop with time. ${ }^{5}$

Radiographically, the luxation of the radial head is usually lateral and caudal to the articular surface of the humerus in

\section{(c) 2022. The Author(s).}

This is an open access article published by Thieme under the terms of the Creative Commons Attribution License, permitting unrestricted use, distribution, and reproduction so long as the original work is properly cited. (https://creativecommons.org/licenses/by/4.0/)

Georg Thieme Verlag KG, Rüdigerstraße 14, 70469 Stuttgart, Germany 
dogs. The radial epiphysis is described as more convex than in unaffected dogs due to the lack of contact with the humerus. Cranial curvature of the proximal ulna, medial deviation and distortion of the olecranon, angular deformity of the distal humerus, hypoplasia of the medial coronoid process and absence of the anconeal process may be added as radiographic findings. $3,7,11$

Reports of the treatment of congenital radial head dislocations in dogs include conservative management, surgical reduction and stabilization, radial head ostectomy and arthrodesis. ${ }^{5,13,15-18}$

To our knowledge, only one report of a cat affected by bilateral CEL type I was diagnosed; however, no surgical correction was attempted. ${ }^{19}$

This case report describes the radial head ostectomy surgery used to treat a cat with congenital radial head luxation and its outcome.

\section{Case Report}

\section{Clinical History}

A 7-month-old female domestic short hair cat was presented for a persistent right forelimb lameness with progressive worsening. The cat had been in possession since it was 2 months old, and no trauma was reported during this time. Subjective evaluation of lameness was performed using the following 5 degrees scoring system. ${ }^{20}$ A grade 3 (persistent moderate weight-bearing lameness) of the right thoracic limb lameness was present, with the tendency not to bear weight on the right forelimb when standing and rotating the elbow internally and externally.

Clinical examination revealed a mild deformation of the forelimb, with the elbow that appeared to bow laterally. On manipulation, the radial head could be easily palpated as it protruded from the lateral humeral condyle in latero-caudal direction and a mild discomfort on manipulation of the elbow was noticed. The elbow's range of motion was restricted, compared with the normal contralateral elbow of 15 degrees in flexion (50 degrees right versus 35 degrees left) and 25 degrees in extension (145 degrees right versus 170 degrees left). No other physical abnormalities of the left forelimb were detected, and the neurologic examinations of both antebrachia revealed no other pathologies.

Cranio-caudal and medio-lateral radiographic views of the left and right forelimbs were obtained under general anaesthesia, including the elbow and carpal joints. The right elbow joint had a caudolateral luxation of the right radial head without disruption of the humeroulnar joint; the ulna presented a recurvatum deformity with abnormal cranial curvature of the medial coronoid process of the ulna (-Fig. 1). The left elbow, radius, and ulna had no abnormalities.

Because of the clinical history, physical examination and radiographic features, type I CEL of the right elbow was diagnosed.

Ostectomy of the radial head was chosen as surgical treatment.

\section{Surgical Planning}

On the preoperative cranio-caudal radiographic view, the osteotomy line was planned to be $1 \mathrm{~cm}$ distal to radial articular surface to eliminate humero-radial conflict.

\section{Surgical Treatment}

Routine haematology, serum biochemistry and electrolytes before surgery were unremarkable. The cat was anaesthetized for surgery. Perioperative analgesia and antibiotic treatment consisted of dexmedetomidine (Dexdor: Orion Corporation, Espoo, Finland, $5 \mu \mathrm{g} / \mathrm{kg}$ ) and ketamine (Ketavet: ACME Srl, Cavriago, Italy, $2 \mathrm{mg} / \mathrm{kg}$ ) that were administered intramuscularly and cefazolin (Cefazolina Dorom: Teva Pharma Italia, Milano, Italy, $22 \mathrm{mg} / \mathrm{kg}$ intravenously) through
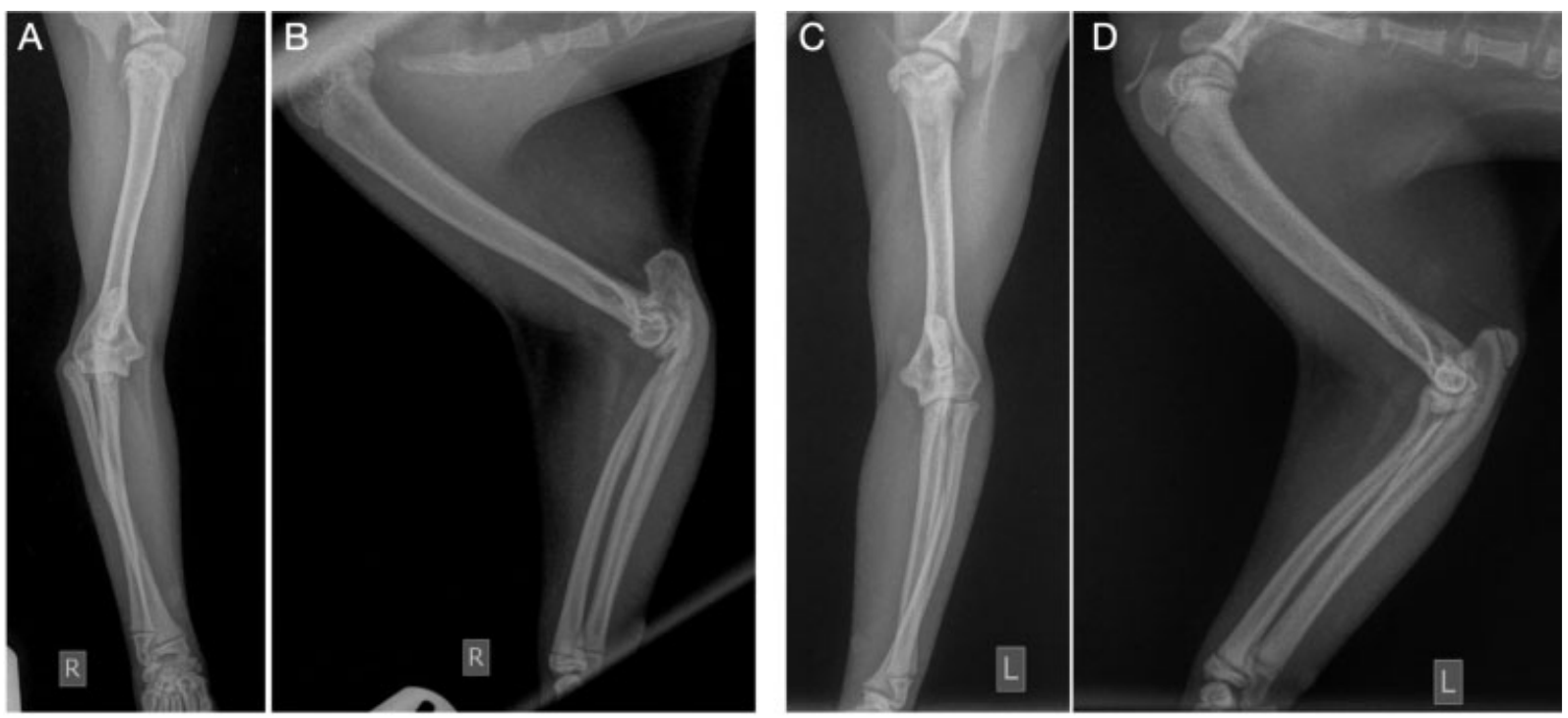

Fig. 1 Cranio-caudal and medio-lateral radiographic views of the right $(A, B)$ and left $(C, D)$ elbow joints. Note the caudolateral sub-luxation of the right radial head without disruption of the humeroulnar joint. The left elbow shows normal anatomic relationship between humerus, radius, and ulna. 
a left cephalic venous catheter. Anaesthesia was induced with propofol (Proposure 1\%: Merial, Italy, $2 \mathrm{mg} / \mathrm{kg}$ intravenously) and maintained with isoflurane (IsoFlo: Aesica Queen-borough Limited, Kent, United Kingdom, 0.5-2\% isoflurane vaporizer setting) in oxygen delivered via a rebreathing circuit with a $0.5 \mathrm{~L} / \mathrm{min}$ oxygen flow. An ultrasoundguided left brachial plexus block was performed with aseptic technique with ropivacaine (Ropivacaina Galenica Senese: Industria Farmaceutica Galenica Senese S.r.l., Siena, Italy, $0.15 \mathrm{~mL} / \mathrm{kg}$ ) before moving to the operating room. Anaesthesia during the surgical procedure was uneventful. Meloxicam (Metacam: Boehringer Ingelheim Vetmedica gmbh, Rhein, Germany, $0.1 \mathrm{mg} / \mathrm{kg}$ ) was administered intravenously at skin closure.

The cat was positioned on the surgical table in left lateral recumbency and prepared for surgery in a hanging limb position. A lateral approach was made over the right radial head, extending from the lateral humeral epicondyle to the proximal third of the radius. ${ }^{21} \mathrm{An}$ incision made between the common and lateral digital extensor muscles was made to reach the joint capsule; a lateral arthrotomy was then performed to expose the radial articular surface. Radial head ostectomy was performed with an osteotome (DePuy Synthes $5 \mathrm{~mm}$ osteotome 399.810) and mallet (DePuy Synthes $300 \mathrm{~g}$ mallet 399.410) extending approximately $1 \mathrm{~cm}$ distal to the joint line. The articular surface of the radial head, which is normally concave, resulted convex because of the altered contact with the opposing articular surface of the humerus. ${ }^{22}$ Lateral collateral ligament integrity was deemed satisfactory by manipulating the extended elbow joint in valgus direction and by performing the Campbell test ${ }^{23}$ (-Fig. 2). Intraoperative evaluation of range of motion revealed an increase in the maximum elbow flexion. The joint and the wound were then routinely closed.

Postoperative radiographs confirmed complete removal of the radial head (-Fig. 3). A Robert-Jones-bandage was applied for 5 days. Postoperative analgesia consisted of buprenorphine (Buprenodale: Dechra Veterinary Products s.r.l., Torino, Italy, $0.02 \mathrm{mg} / \mathrm{kg}$ intramuscularly every
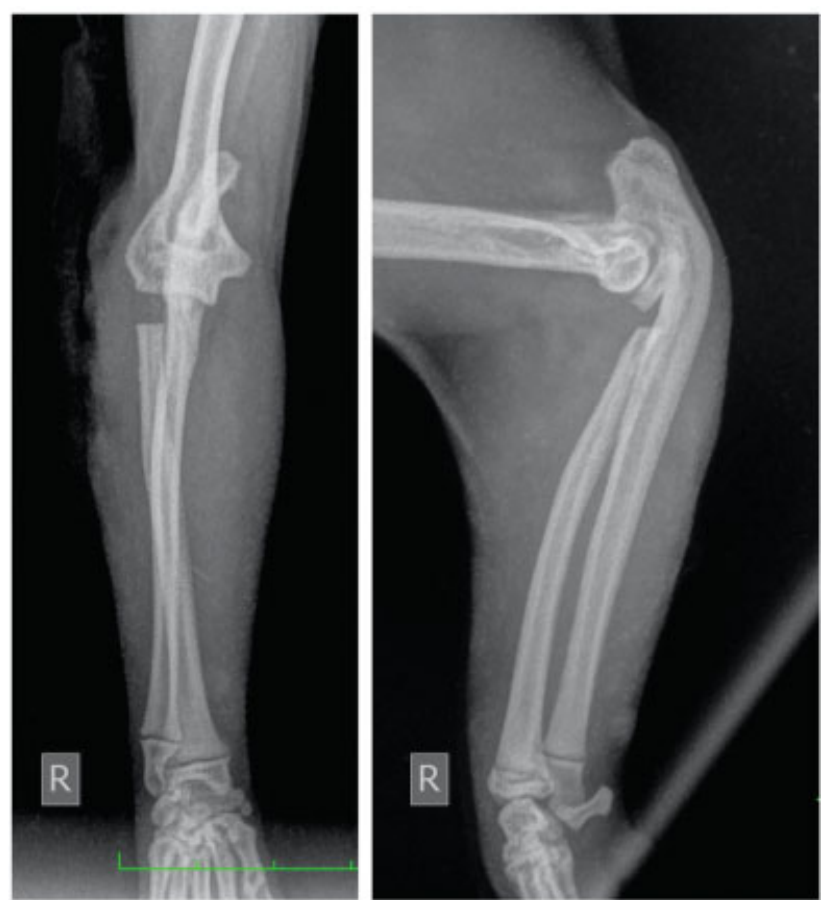

Fig. 3 Immediate postoperative cranio-caudal and medio-lateral radiographic views of the right elbow showing complete removal of the radial head.

8 hours) for 24 hours and non-steroidal anti-inflammatory drug analgesia was continued for 7 days (Meloxicam, 0.05 $\mathrm{mg} / \mathrm{kg}$ orally once daily).

Cage-rest was recommended for 2 weeks, followed by in house confinement for other 2 weeks.

Re-examination 4 weeks after the surgery showed a grade $3 / 5$ right thoracic limb lameness, ${ }^{20}$ with light pain on palpation and manipulation of the right elbow. The elbow joint's range of motion was equivalent to the contralateral elbow.

Re-evaluation 2 months after surgery showed a lameness grade improvement of $2 / 5$ and no pain was induced by the manipulation of the articulation of the elbow. Radiographic images documented a remodelling of the radial head toward

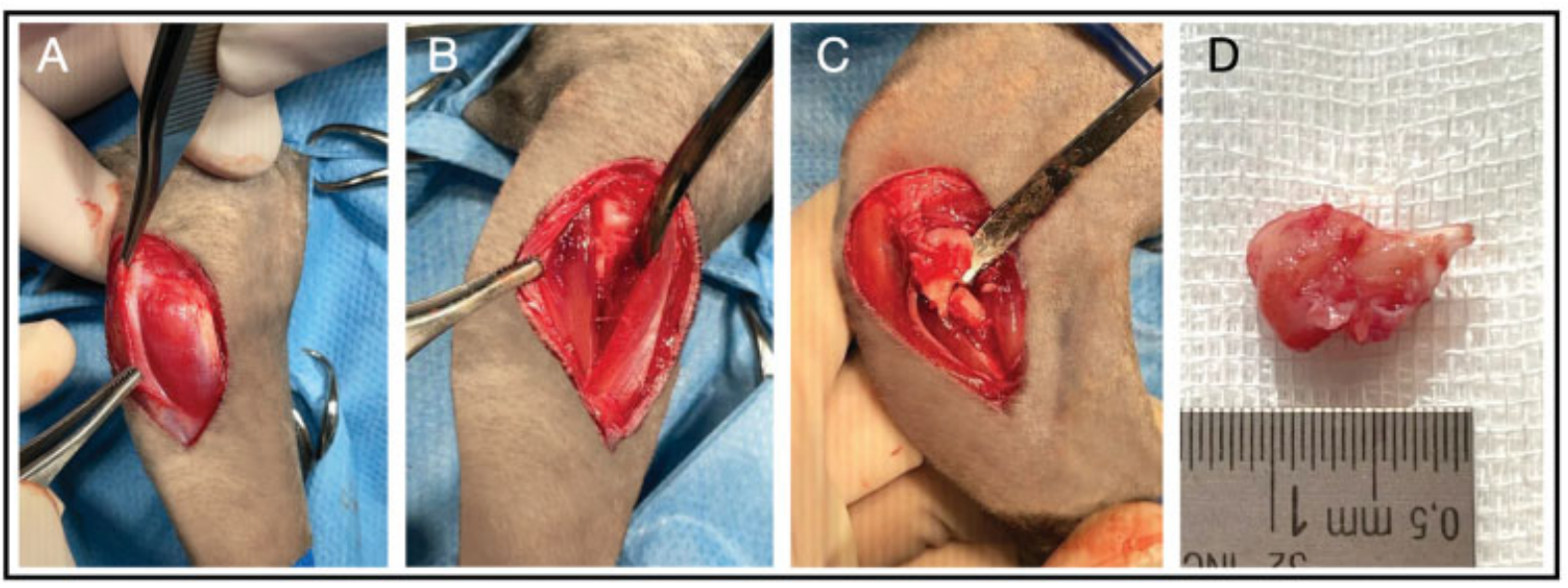

Fig. 2 Intraoperative images. The subluxated radial head is protruding under the common and lateral digital extensor muscles (A). Dissection between those muscles allowed exposure of radial head (B) and performing of the ostectomy (C). According to the preoperative planning, in order to eliminate humero-radial conflict, the excised radius fragment measured about $1 \mathrm{~cm}$ (D). 

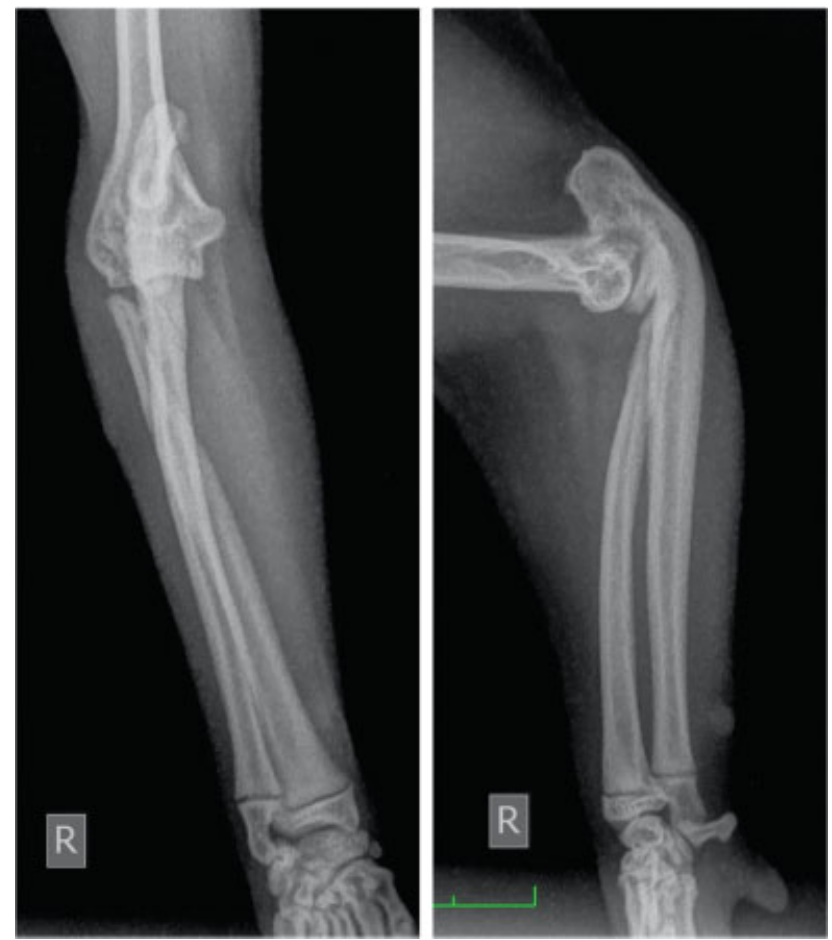

Fig. 4 Cranio-caudal and medio-lateral radiographic views of the right elbow taken two months postoperatively: note the remodelling of proximal towards the lateral condyle of the humerus because of the growth of the distal radial physis.

the lateral condyle of the humerus, without bone contact and without progression of joint degeneration (-Fig. 4). Exercise restriction was relaxed.

The last evaluation at 4 months after surgery showed a complete resolution of the lameness (grade 1 ) and no pain on manipulation and palpation of the right elbow. Radiographic assessment confirmed no signs of degenerative disease, and the radial osteotomy line appears to have progressed in a proximal direction, but without lateral subluxation and without bone conflict with the condyle of the humerus (-Fig. 5).

\section{Discussion}

Congenital luxation of the radial head of cats is a rare condition that lacks description of a consistent technique for surgical treatment. The limited reports about similar congenital defects in dogs, of the surgical techniques, follow-up periods and complications make clinical and surgical decision-making, especially in a different species, more challenging. ${ }^{5,11,13,15-17}$ It is still debated whether and when to attempt surgical intervention. Dogs that show mild clinical signs should be managed with a conservative approach and they should be strictly monitored with radiographies until the development of pain or progressive subluxation of the elbow warrant surgical intervention. ${ }^{18}$ However, whenever a preventive approach is preferred, a surgical correction should be performed as early as possible to reduce the risk of secondary osteoarthrosis and to allow a better remodelling of the humero-radial articulation. ${ }^{10,18,24}$ In-

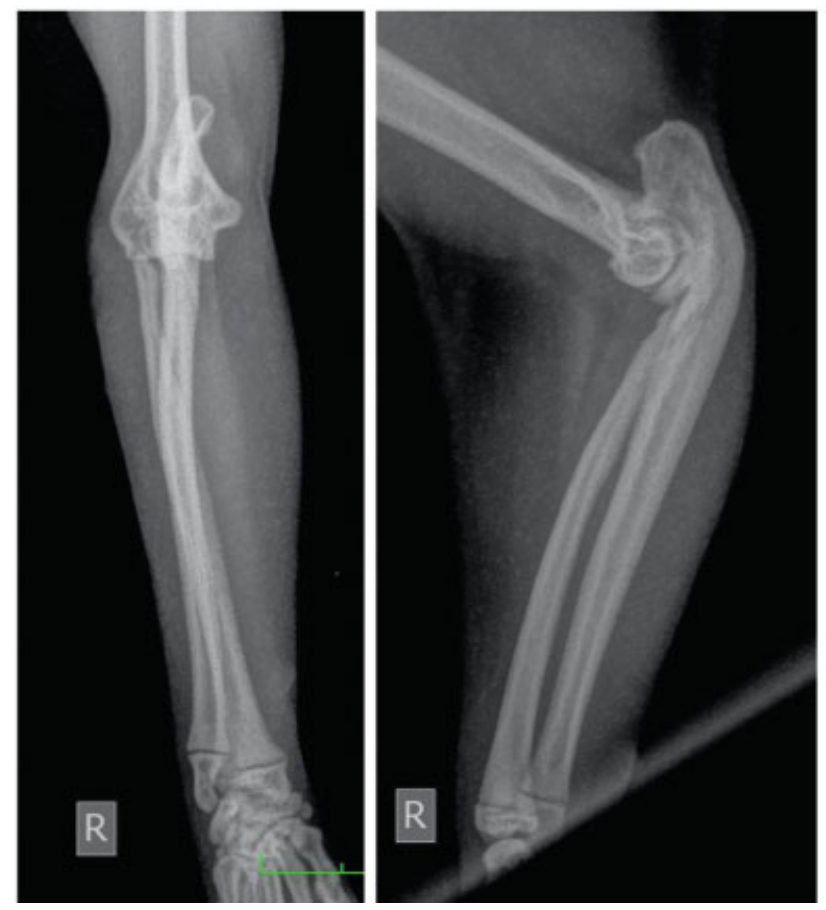

Fig. 5 Cranio-caudal and medio-lateral radiographic views of the right elbow at 4 months postoperative recheck. No signs of degenerative disease are evident. The radial osteotomy line appears to have progressed in a proximal direction, but without lateral subluxation and without bone conflict with the condyle of the humerus.

deed, the reduction in the radial head before 5 months of age will result in better remodelling of the articular cartilage than if surgery is delayed. ${ }^{14,24}$ Based on theorical predictive surgery's data in dogs, the same criteria may be applied to cats; thus, preventive therapy was chosen for this clinical case, with the assumption that a persistent deformity, a longterm lameness, restricted range of motion and a permanent dislocation will lead to osteoarthrosis. ${ }^{16}$ The surgical procedure of radial head-ostectomy was chosen as it was found to be easier to apply, inexpensive and with less demanding postoperative care compared with radial head reduction techniques. ${ }^{13,15,17,18,25}$ Reduction in radial head is supposed to allow better clinical function because of restoration of anatomical relationship between the radius and humerus. In children managed surgically for chronic post-traumatic radial head luxation, a good prognosis depends upon the presence of a normal concave radial head articular surface and is not dependent on the age of the patient and duration of the dislocation per se. ${ }^{26}$ Radial head ostectomy was preferred to surgical reduction in the present case as the latter was deemed impossible because of the chronicity of the lesion with bone remodelling, particularly the excessive length of the radius and the recurvatum deformity of the ulna. Moreover, the altered convex surface of radial head because of lack of contact with the opposing humeral articular cartilage surface would have created a misarticulation of the incongruent humeroradial joint surfaces. Furthermore, the radial head ostectomy avoids potential complications of implant use (external fixator, transarticular 
pins or screws) such as damage to the growth plates or implant breakage or migration. ${ }^{14,16}$

Additionally, reconstruction or augmentation of the lateral collateral ligament was not attempted because of the good stability of the elbow joint examined during surgery after the ostectomy, probably caused by the compensation of remodelling of the ulnar coronoid processes that fully articulate with the humerus.

The level of ostectomy line was estimated to avoid that the remaining growth potential of the distal radial growth plate could result in a recurrence of conflict between the proximal radius and humeral condyle. Radiographs at 4 months postoperatively confirmed remodelling of the ostectomy line in proximal direction, without humero-radial conflict. Considering that age of the cat at time of the last recheck was 11 months, it is presumable that the residual growth potential will not cause any substantial continued proximal progression of the radial stump.

To our knowledge, no reports on the surgical treatment of CEL type I in cats were hitherto described; therefore, the treatment by radial head-ectomy in this single case does not allow any further conclusions except that in our case the procedure was effective and of satisfactory outcome. The relative short follow-up time remains a limitation of this report in terms of long-term function of the joint.

\section{Authors' Contribution}

All authors contributed to management of the case, acquisition of data, drafting, and revision of the manuscript.

\section{Funding}

None.

\section{Conflict of Interests}

None.

\section{References}

1 Campbell JR. Nonfracture injuries to the canine elbow. J Am Vet Med Assoc 1969;155(05):735-744

2 Milton JL, Montgomery RD. Congenital elbow dislocations. Vet Clin North Am Small Anim Pract 1987;17(04):873-888

3 Kene ROC, Lee R, Bennett D. The radiological features of congenital elbow luxation/subluxation in the dog. J Small Anim Pract 1982; 23:621-630

4 Evans HE, Christiensen GC. In: Evans HE, Christiensen GC, eds. Appendicular skeleton. Miller's Anatomy of the Dog. 2nd edition. Philadelphia: W.B. Saunders Company; 1979499

5 Griffon DJ. Surgical diseases of the elbow. In: Tobias KM, Johnson SA, eds. Veterinary Surgery Small Animal. 1st edition. St. Louis: W. B. Saunders; 2012:730-732

6 Grøndalen J. Malformation of the elbow joint in an Afghan hound litter. J Small Anim Pract 1973;14(02):83-89

7 Stevens DR, Sande RD. An elbow dysplasia syndrome in the dog. J Am Vet Med Assoc 1974;165(12):1065-1069
8 Bingel SA, Riser WH. Congenital elbow luxation in the dog. J Small Anim Pract 1977;18(07):445-456

9 Milton JL, Horne RD, Bartels JE, Henderson RA. Congenital elbow luxation in the dog. J Am Vet Med Assoc 1979;175(06):572-582

10 Gurevitch R, Hohn RB. Surgical management of lateral luxation and subluxation of the canine radial head. Vet Surg 1980;9:49-57

11 Campbell JR. Congenital luxation of the elbow of the dog. Veterinary Annual 1979;19:229-236

12 Temwichitr J, Leegwater PAJ, Auriemma E, et al. Evaluation of radiographic and genetic aspects of hereditary subluxation of the radial head in Bouviers des Flandres. Am J Vet Res 2010;71(08): 884-890

13 Fafard AR. Unilateral congenital elbow luxation in a dachshund. Can Vet J 2006;47(09):909-912

14 Clark KJ, Jerram RM, Walker AM. Surgical management of suspected congenital luxation of the radial head in three dogs. N Z Vet J 2010;58(02):103-109

15 Spardari A, Romagnoli N, Venturini A. A modified Bell-Tawse proce_dure for surgical correction of congenital elbow luxation in a Dalmation puppy. Vet Comp Orthop Traumatol 2001; $14: 210-213$

16 Fitzpatrick N, Yeadon R, Farrell M. Surgical management of radial head luxation in a dog using an external skeletal traction device. Vet Comp Orthop Traumatol 2013;26(02):140-146

17 Heidenreich DC, Fourie Y, Barreau P. Presumptive congenital radial head sub-luxation in a shih tzu: successful management by radial head ostectomy. J Small Anim Pract 2015;56(10): 626-629

18 Dassler C, Vasseur PB. Elbow luxation. In: Textbook of Small Animal Surgery. 3rd edition Philadelphia: W.B. Saunders Company; 2003:1919-1927

19 Valastro C, Di Bello A, Crovace A. Congenital elbow subluxation in a cat. Vet Radiol Ultrasound 2005;46(01):63-64

20 Skaggs S, DeAngelis MP, Rosen H. Deformities due to premature closure of the distal ulna in fourteen dogs. J Am Anim Hosp Assoc 1973;9:496-500

21 Piermattei DL, Johnson KA. Approach to the head and proximal metaphysis of the radius. In: Piermattei DL, Johnson KA, eds. An Atlas of Surgical Approaches to the Bones and Koints of the Dog and Cat. 4th edition. Philadelphia: WB Saunders Company; 2004: 242-245

22 Fox DJ. Radius and ulna. In: Tobias KM, Johnston SA, eds. Veterinary Surgery Small Animal. 1st edition. W. B. Saunders; 2012: 760-784

23 Farrell M, Draffan D, Gemmill T, Mellor D, Carmichael S. In vitro validation of a technique for assessment of canine and feline elbow joint collateral ligament integrity and description of a new method for collateral ligament prosthetic replacement. Vet Surg 2007;36(06):548-556

24 Piermattei DL, Flo GL, DeCamp CE. Congenital luxation of the radial head. In: Brinker, Piermattei and Flo's Handbook of Small Animal Orthopedics and Fracture Repair. 4th edition. Missouri: Saunders Elsevier; 2006:336-339

25 Fitzpatrick N. Radial head transfer for congenital elbow luxation: Surgical treatment of congenital and developmental luxations of the radial head. Paper presented at: American College of Veterinary Surgery Symposium; October 23-25, 2008San Diego, USA

26 Seel MJ, Peterson HA. Management of chronic posttraumatic radial head dislocation in children. J Pediatr Orthop 1999;19 (03):306-312 